\title{
KAM theorem for the nonlinear Schrödinger equation
}

\author{
Grébert, B ; Kappeler, T
}

\begin{abstract}
We prove the persistence of finite dimensional invariant tori associated with the dfocusing nonlinear Schrödinger equation under small Hamiltonian perturbations. The invariant tori are not necessarily small.
\end{abstract}

DOI: https://doi.org/10.2991/jnmp.2001.8.s.23

Other titles: Nonlinear evolution equations and dynamical systems (Kolimbary, 1999)

Posted at the Zurich Open Repository and Archive, University of Zurich ZORA URL: https://doi.org/10.5167/uzh-22028 Journal Article

Originally published at:

Grébert, B; Kappeler, T (2001). KAM theorem for the nonlinear Schrödinger equation. Journal of Nonlinear Mathematical Physics, 8(suppl.):133-138.

DOI: https://doi.org/10.2991/jnmp.2001.8.s.23 


\section{KAM Theorem for the Nonlinear Schrödinger Equation}

Benoît GRÉBERT ${ }^{\dagger}$ and Thomas KAPPELER $\ddagger$

† Département de Mathématiques, UMR 6629, Université de Nantes 2 rue de la Houssinière, BP 92208, 44322 Nantes cedex 3 France

E-mail: grebert@math.univ-nantes.fr

‡ Institut für Mathematik, Universität Zürich, Winterthurerstrasse 190 CH-8057 Zürich, Switzerland

E-mail: tk@math.unizh.ch

\section{Abstract}

We prove the persistence of finite dimensional invariant tori associated with the defocusing nonlinear Schrödinger equation under small Hamiltonian perturbations. The invariant tori are not necessarily small.

\section{Introduction}

Consider the defocusing nonlinear Schrödinger equation on a circle of unit length,

$$
i \partial_{t} \varphi=-\partial_{x x} \varphi+2|\varphi|^{2} \varphi \quad\left(t \in \mathbb{R}, x \in S^{1}\right) .
$$

It is a completely integrable Hamiltonian system of infinite dimension with phase space $H^{N} \equiv H^{N}\left(S^{1}, \mathbb{C}\right)\left(N \in \mathbb{R}_{\geq 1}\right)$ and Hamiltonian $H_{0} \equiv H_{0}(\varphi, \bar{\varphi})$. Here

$$
H^{N}\left(S^{1}, \mathbb{C}\right)=\left\{f(x)=\sum_{k \in \mathbb{Z}} \hat{f}(k) e^{2 i \pi k x} \mid\|f\|_{N}<\infty\right\},
$$

where

$$
\|f\|_{N}^{2}:=\sum_{k \in \mathbb{Z}}(1+|k|)^{2 N}|\hat{f}(k)|^{2}
$$

and

$$
H_{0}(\varphi, \bar{\varphi}):=\int_{0}^{1}\left(\left|\varphi_{x}\right|^{2}+|\varphi|^{4}\right) d x .
$$

The Poisson structure is given by the regular Poisson bracket

$$
\{F, G\}:=i \int_{S^{1}}\left(\frac{\partial F}{\partial \varphi(x)} \frac{\partial G}{\partial \bar{\varphi}(x)}-\frac{\partial F}{\partial \bar{\varphi}(x)} \frac{\partial G}{\partial \varphi(x)}\right) d x
$$


where $F, G$ are functionals on $L^{2} \equiv L^{2}\left(S^{1}, \mathbb{C}\right)$ of class $C^{1}$. When written as a Hamiltonian system, (1) takes the form

$$
\partial_{t} \varphi=\left\{H_{0}, \varphi\right\}=-i \frac{\partial H_{0}}{\partial \bar{\varphi}} .
$$

Our aim is to prove the existence of quasiperiodic solutions, not necessarily small, of small Hamiltonian perturbations of (2), i.e. of the equation

$$
\partial_{t} \varphi=-i \frac{\partial H}{\partial \bar{\varphi}}
$$

where

$$
H(\varphi, \bar{\varphi})=H_{0}(\varphi, \bar{\varphi})+\varepsilon K(\varphi, \bar{\varphi}), \quad \varepsilon \quad \text { small }
$$

To obtain solutions which are not necessarily close to zero we use a method developed in [11] for the $\mathrm{KdV}$ equation: First we prove the existence of global Birkhoff variables $\left(x_{j}, y_{j}\right)_{j \in \mathbb{Z}}$ (see Section 2). In these new variables, the NLS equation takes the canonical form

$$
\left\{\begin{array}{l}
\dot{x}_{k}=-w_{k} y_{k}, \\
\dot{y}_{k}=w_{k} x_{k},
\end{array}\right.
$$

where $\left({ }^{*}\right)$ denotes the time derivative and $w(I)=\left(w_{k}(I)\right)_{k \in \mathbb{Z}}$ is the sequence of frequencies which depend only on the actions $I_{j}=\left(x_{j}^{2}+y_{j}^{2}\right) / 2, j \in \mathbb{Z}$.

We then verify non-resonance conditions for the frequencies of the unperturbed system (2) reduced by certain symmetries which allow us to apply a refined version [15] of a KAM-theorem of Kuksin [12].

The results of this work have been anounced in [6] and proved in a series of articles $[7,8,9]$ and $[10]$.

\section{Existence of global Birkhoff variables}

It is well known that NLS admits a Lax pair representation

$$
\frac{\partial L}{\partial t}=[L, M]:=L M-M L
$$

where

$$
L=L(\varphi):=i\left(\begin{array}{rr}
1 & 0 \\
0 & -1
\end{array}\right) \frac{d}{d x}+\left(\begin{array}{cc}
0 & \varphi \\
\bar{\varphi} & 0
\end{array}\right)
$$

is the Zakharov-Shabat operator and $M$ is a rather complicated operator given in [4]. As a consequence, the periodic spectrum of $L(\varphi), \operatorname{spec}(\varphi):=\left\{\lambda \in \mathbb{C}, \exists F \in H_{\mathrm{loc}}^{1}\left(\mathbb{R}, \mathbb{C}^{2}\right)\right.$ with $F \neq 0, L(\varphi) F=\lambda F$ and $F(x+2)=F(x), x \in \mathbb{R}\}$, remains invariant under the NLS flow. The periodic spectrum consists of two interlacing sequences $\left(\lambda_{k}^{+}(\varphi)\right)_{k \in \mathbb{Z}},\left(\lambda_{k}^{-}(\varphi)\right)_{k \in \mathbb{Z}}$ of real numbers satisfying $\lambda_{k}^{ \pm} \sim k \pi\left(|k|\right.$ large) and $\lambda_{k}^{-} \leq \lambda_{k}^{+}<\lambda_{k+1}^{-}, k \in \mathbb{Z}$ (cf [14], [5]). Furthermore, $\operatorname{spec}(\varphi)$ is a complete set of integrals for the NLS equation (cf. [4]). This fact is used to prove the following Theorem (cf. [1] and [7]), 
Theorem 1. For any $N \geq 1$, there exists a bianalytic, bijective symplectomorphism

$$
\Phi: l_{N}^{2}\left(\mathbb{Z}, \mathbb{R}^{2}\right) \rightarrow H^{N}\left(S^{1}, \mathbb{R}^{2}\right)
$$

such that $\left(x_{j}, y_{j}\right)_{j \in \mathbb{Z}}=\Phi^{-1}(\varphi)$ are Birkhoff coordinates for $N L S$, i.e. $I_{j}=\frac{1}{2}\left(x_{j}^{2}+y_{j}^{2}\right)$ are action and $\theta_{j}=\operatorname{arctg}\left(\frac{y_{j}}{x_{j}}\right)$ are angle variables.

Here

$$
l_{N}^{2}\left(\mathbb{Z}, \mathbb{R}^{2}\right):=\left\{\left(a_{j}, b_{j}\right)_{j \in \mathbb{Z}},\left\|\left(a_{j}\right)_{j \in \mathbb{Z}}\right\|_{N}+\left\|\left(b_{j}\right)_{j \in \mathbb{Z}}\right\|_{N}<+\infty\right\},
$$

where

$$
\left\|\left(a_{j}\right)_{j \in \mathbb{Z}}\right\|_{N}^{2}=\sum_{j \in \mathbb{Z}}(1+|j|)^{2 N}\left|a_{j}\right|^{2}
$$

and $l_{N}^{2}\left(\mathbb{Z}, \mathbb{R}^{2}\right)$ is endowed with the canonical symplectic structure

$$
\Omega\left(\left(a_{j}, b_{j}\right)_{j \in \mathbb{Z}},\left(x_{j}, y_{j}\right)_{j \in \mathbb{Z}}\right)=2 \sum_{j \in \mathbb{Z}} a_{j} y_{j}-b_{j} x_{j} .
$$

In action angle variables, the Hamiltonian $H_{0}$ depends only on the actions, $H_{0}(\varphi, \bar{\varphi})=$ $\mathcal{H}(I)$, and NLS is equivalent to the system (5), where $w_{k}(I)=\frac{\partial \mathcal{H}}{\partial I_{k}}(I)$.

In particular, given $I \in l_{2 N}^{1}\left(\mathbb{Z}, \mathbb{R}_{+}\right)$with finite support,

$$
\mathcal{T}_{I}=\Phi\left(\left\{\left(x_{j}, y_{j}\right)_{j \in \mathbb{Z}}, x_{j}^{2}+y_{j}^{2}=2 I_{j}, j \in \mathbb{Z}\right\}\right)
$$

is an invariant set diffeomorphic to a torus whose dimension is $\sharp\left\{k \in \mathbb{Z}, I_{k} \neq 0\right\}$.

The solution $\phi(x, t) \equiv \varphi_{t}(x)$ of the initial value problem for NLS, with initial profile $\varphi_{0}=\Phi\left(\left(\sqrt{2 I_{j}} e^{i \theta_{j}}\right)_{j \in \mathbb{Z}}\right)$ in $H^{N}\left(S^{1}, \mathbb{C}\right)$, is given by

$$
\varphi_{t}=\Phi\left(\left(\sqrt{2 I_{j}} e^{i\left(\theta_{j}+t w_{j}(I)\right)}\right)_{j \in \mathbb{Z}}\right) .
$$

\section{KAM Theorem for NLS}

An asymptotic expansion shows that the frequencies have asymptotic resonances,

$$
w_{ \pm k}(I) \sim 4 \pi^{2} k^{2} \quad \text { for } \quad k \quad \text { large. }
$$

In order to control their effect on perturbed equations we impose symmetry conditions on the perturbation. These conditions (see [8]) allow to consider as phase spaces the subspaces $H_{\alpha}^{N}\left(S^{1}, \mathbb{C}\right), \alpha \in \mathbb{R}$, defined by,

$$
H_{\alpha}^{N}\left(S^{1}, \mathbb{C}\right):=\Phi\left(l_{N ; \alpha}^{2}\left(\mathbb{Z}, \mathbb{R}^{2}\right)\right),
$$

where $\left(\sqrt{2 I_{j}} e^{i \theta_{j}}\right)_{j \in \mathbb{Z}} \in l_{N ; \alpha}^{2}$ iff $\left(\sqrt{2 I_{j}} e^{i \theta_{j}}\right)_{j \in \mathbb{Z}} \in l_{N}^{2}$ and satisfies

$$
I_{-j}=I_{j} \quad \forall j \geq 1,
$$


and

$$
\theta_{-j} \equiv \theta_{j}+\alpha(\bmod 2 \pi) \quad \forall j \geq 0 \quad \text { with } \quad I_{j} \neq 0 .
$$

(Notice that for $\alpha \not \equiv 0(\bmod 2 \pi),(11)$ implies that $I_{0}(\varphi)=0$ for all $\varphi \in H_{\alpha}^{N}$.)

One verifies that the subspaces $H_{\alpha}^{N}\left(S^{1}, \mathbb{C}\right)$ are invariant under the NLS-flow by showing (cf. [9] ) that the symmetries of the NLS Hamiltonian $\mathcal{H}(I)$ imply that $\mathcal{H}(\mathcal{J}(I))=\mathcal{H}(I)$, where $\mathcal{J}(I)_{k}=I_{-k} \forall k \in \mathbb{Z}$. As a consequence, the frequencies $w_{j}=\frac{\partial \mathcal{H}}{\partial I_{j}}$ are symmetric at points where $\mathcal{J}(I)=I$.

Moreover, in [8] we provide the following characterization of $H_{\alpha}^{N}\left(S^{1}, \mathbb{C}\right)$,

$$
H_{\alpha}^{N}\left(S^{1}, \mathbb{C}\right)=\left\{\varphi \in H^{N}\left(S^{1}, \mathbb{C}\right) \mid e^{i \alpha} \check{\varphi} \equiv \varphi\right\},
$$

where $\check{\varphi}(x)=\varphi(-x)$. In particular, $H_{\pi}^{N} \cap C^{\infty}\left(\right.$ resp. $\left.H_{0}^{N} \cap C^{\infty}\right)$ is the phase-space of $\varphi \in H^{N} \cap C^{\infty}$ satisfying generalized Dirichlet (resp. Neumann) conditions, i.e. $\partial_{x}^{2 k} \varphi(0)=$ $\partial_{x}^{2 k} \varphi(1)=0\left(\right.$ resp. $\left.\partial_{x}^{2 k+1} \varphi(0)=\partial_{x}^{2 k+1} \varphi(1)=0\right) \forall k \in \mathbb{Z}$. By a slight abuse of notation, the restriction of $\Phi$ to $l_{N, \alpha}^{2}\left(\mathbb{Z}, \mathbb{R}^{2}\right)$ is again denoted by $\Phi$.

For $\alpha \in \mathbb{R} / 2 \pi \mathbb{Z}$, a finite subset $A \subseteq \mathbb{Z}_{\geq 0}$ (with $0 \notin A$ if $\alpha \not \equiv 0$ ) and $I_{A} \in\left(\mathbb{R}_{>0}\right)^{|A|}$ we denote by $T_{I_{A}}^{\alpha}$ the $|A|$ dimensional torus of the model space $l^{2}\left(\mathbb{Z} ; \mathbb{R}^{2}\right)$, defined by

$$
\begin{aligned}
T_{I_{A}}^{\alpha}:= & \left(\sqrt{2 J_{j}} e^{i \theta_{j}}\right)_{j \in \mathbb{Z}} \mid J_{j}=J_{-j}=I_{j}, \forall j \in A ; \\
& \left.J_{j}=J_{-j}=0, \forall j \notin A ; \theta_{j}=\theta_{-j}+\alpha, \forall j \in A\right\}
\end{aligned}
$$

and by $\mathcal{T}_{I_{A}}^{\alpha}$ the $|A|$ dimensional torus in $H_{\alpha}^{N}$, invariant under NLS,

$$
\mathcal{T}_{I_{A}}^{\alpha}:=\Phi\left(T_{I_{A}}^{\alpha}\right) .
$$

For $\Gamma \subseteq\left(\mathbb{R}_{>0}\right)^{|A|}$ compact and of positive Lebesgue measure, introduce

$$
\mathcal{T}_{\Gamma}^{\alpha}:=\cup_{I_{A} \in \Gamma} \mathcal{T}_{I_{A}}^{\alpha} .
$$

The set $\mathcal{T}_{\Gamma}^{\alpha}$ consists of symmetric $2|A|$-gap potentials (if $0 \notin A$ ) or $(2|A|-1$ )-gap potentials (if $0 \in A$ ), i.e potentials $\varphi \in H_{\alpha}^{0}$ with $\lambda_{j}^{+}(\varphi) \neq \lambda_{j}^{-}(\varphi)$ iff $|j| \in A$ and $\lambda_{-j}^{+}(\varphi)-\lambda_{-j}^{-}(\varphi)=$ $\lambda_{j}^{+}(\varphi)-\lambda_{j}^{-}(\varphi) \forall j \geq 1$ (cf. [8]).

We consider Hamiltonian perturbations, $H_{\varepsilon}=H_{0}+\varepsilon K$ on $H_{\alpha}^{N}\left(S^{1}, \mathbb{C}\right)$ with the following properties:

(P1) $K$ is real analytic on some symmetric neighborhood $U_{\Gamma}$ of $\left\{(\varphi, \bar{\varphi}), \varphi \in \mathcal{T}_{\Gamma}^{\alpha}\right\}$ in $\left(H^{N}\left(S^{1}, \mathbb{C}\right)\right)^{2}$.

(P2) $\frac{\partial K}{\partial \varphi}, \frac{\partial K}{\partial \psi}$ are bounded as functions from $U_{\Gamma}$ into $H^{N}\left(S^{1}, \mathbb{C}\right)$ and verify the normalization condition

$$
\sup \left\{\left\|\frac{\partial K}{\partial \varphi}(\varphi, \psi)\right\|_{N}+\left\|\frac{\partial K}{\partial \psi}(\varphi, \psi)\right\|_{N} \mid(\varphi, \psi) \in U_{\Gamma}\right\} \leq 1 .
$$

(P3) $K$ satisfies the symmetry condition, $\left((\varphi, \psi) \in U_{\Gamma}\right)$

$$
K(\varphi, \psi)=K\left(e^{i \alpha} \check{\varphi}, e^{-i \alpha} \check{\psi}\right)
$$

\footnotetext{
${ }^{1} U_{\Gamma}$ is said to be symmetric iff $\left(e^{i \alpha} \check{\varphi}, e^{-i \alpha} \check{\psi}\right) \in U_{\Gamma}$ for any $(\varphi, \psi) \in U_{\Gamma}$.
} 
Notice that condition (P3) insures that solutions of $\frac{\partial \varphi}{\partial t}=i \frac{\partial H_{\varepsilon}}{\partial \varphi}$ for initial data in $H_{\alpha}^{N}\left(S^{1}, \mathbb{C}\right)$ evolve in the same space $H_{\alpha}^{N}\left(S^{1}, \mathbb{C}\right)$.

Our KAM Theorem states that, for $\varepsilon$ small enough, many of the NLS-invariant tori $\mathcal{T}_{I_{A}}^{\alpha}$ persist under perturbation of the NLS Hamiltonian by $\varepsilon K$ with $K$ satisfying (P1), (P2), and (P3). Moreover these tori and their linear flows are only slightly perturbed.

Denote by $T^{n}$ the $n$-dimensional torus $(\mathbb{R} / \mathbb{Z})^{n}$.

Theorem 2. Let $N \geq 1, A, \Gamma, \alpha, U_{\Gamma}$ be given as above. Then, for $K$ satisfying (P1), (P2) and (P3), there exists $\varepsilon_{0}$ so that for any $\varepsilon$ with $|\varepsilon| \leq \varepsilon_{0}$ the following statements hold:

(i) there exists a Cantor set $\Gamma_{\varepsilon} \subset \Gamma$ with meas $\left(\Gamma \backslash \Gamma_{\varepsilon}\right) \stackrel{\varepsilon \rightarrow 0}{\rightarrow} 0$,

(ii) there exists a Lipschitz family of real analytic torus embeddings

$$
\Psi: T^{|A|} \times \Gamma_{\varepsilon} \rightarrow U_{\Gamma} \cap\left\{(\varphi, \bar{\varphi}) \mid \varphi \in H_{\alpha}^{N}\right\}
$$

and

(iii) there exists a Lipschitz map $f: \Gamma_{\varepsilon} \rightarrow \mathbb{R}^{|A|}$

such that for $I_{A} \in \Gamma_{\varepsilon}$ and $\theta_{A} \in T^{|A|}, \Psi\left(\theta_{A}+t f\left(I_{A}\right), I_{A}\right)$ is a quasiperiodic solution of $\partial_{t} \varphi=i \frac{\partial H_{0}}{\partial \bar{\varphi}}+i \varepsilon \frac{\partial K}{\partial \bar{\varphi}}$. Moreover, the deformed invariant tori, $\Psi\left(T^{|A|} \times\left\{I_{A}\right\}\right)$, are linearly stable.

\section{Remarks:}

1. Theorem 2 generalizes results due to Kuksin-Pöschel [13] which concern the special case where $\Gamma \subseteq \mathbb{R}_{+}^{|A|}$ is contained in a sufficiently small neighbourhood of $0 \in \mathbb{R}^{|A|}$ and the phase space consists of elements satisfying generalized Dirichlet boundary conditions. In this situation, action-angle variables are not needed as the Fourier coefficients $(\hat{\varphi}(k))_{k \in \mathbb{Z}}$ are a sufficiently good approximation of the Birkhoff coordinates close to the origin.

2. Similarly, the results of [3] and their generalization in [2], while not directly comparable with our Theorem 2, concern only small perturbations of NLS around $\varphi=0$ as well.

3. Our results and methods continue the investigation in [11] on the Korteweg-de Vries equation. The purpose of this paper is to document similar features of the NLS equation.

\section{References}

[1] Bättig D, Bloch A, Guillot J C and Kappeler T, On the Symplectic Structure of the Phase Space for Periodic KdV, Toda and Defocusing NLS, Duke Math. J., 1995, V.79, 549-604.

[2] Bourgain J, Nonlinear Schrödinger Equations, Preprint, Princeton.

[3] Craig W and Wayne E, Periodic Solutions of Nonlinear Schrödinger Equations and NashMoser Method, Preprint, 1993.

[4] Faddeev L D and Takhtajan L A, Hamiltonian Methods in the Theory of Solitons, Springer Verlag, 1987.

[5] Grébert B and Guillot J C, Gaps of one Dimensional Periodic AKNS Systems, Forum Math., 1993, V.5, 459-504. 
[6] Grébert B and Kappeler T, Théorème de Type KAM Pour L'équation de Schrödinger Non Linéaire, C. R. Acad. Sci. Paris, t. 327, Série 1, 1998, 473-478.

[7] Grébert B and Kappeler T, Birkhoff Coordinates for the Nonlinear Schrödinger Equation (in preparation).

[8] Grébert B and Kappeler T, Symmetries of the Nonlinear Schrödinger Equation, Preprint.

[9] Grébert B and Kappeler T, Perturbations of the Defocusing NLS Equation, Preprint.

[10] Grébert B, Kappeler T and Mityagin B, Gap Estimates of the Spectrum of the ZakharovShabat System, Appl. Math. Lett., 1998, V.11, N 4, 95-97.

[11] Kappeler T and Pöschel J, Perturbations of KdV Equations, Preprint, 1997.

[12] Kuksin S, Nearly Integrable Infinite-Dimensional Hamiltonian Systems, LNM 1556, Springer, 1993.

[13] Kuksin S and Pöschel J, Invariant Cantor Manifolds of Quasi-Periodic Oscillations for a Non Linear Schrödinger Equation, Ann. Math., 1996, V.143, 149-179.

[14] Misyura T V, Properties of the Spectra of Periodic and Antiperiodic Boundary Value Problems Generated by Dirac Operators I, II, Theor. Funktsii Funksional. Anal. i Prilozhen, 1978, V.30, 90-101; 1979, V.31, 102-109 (in Russian).

[15] Pöschel J, A KAM Theorem for Some Nonlinear Partial Differential Equations, Ann. Sc. Norm. Sup. Pisa, 1996, V.23, 119-148. 